\title{
Utility of glypican-3 in differentiating hepatocellular carcinoma from other primary and metastatic lesions in FNA of the liver: an immunocytochemical study
}

\author{
Saverio Ligato, Daniza Mandich and Richard W Cartun \\ Department of Pathology and Laboratory Medicine, Hartford Hospital, Hartford, CT, USA
}

\begin{abstract}
We evaluated the immunocytochemical expression of GPC3 in archival material obtained from fine needle aspiration of hepatic lesions to assess the sensitivity and specificity of this marker in cytological material and its potential diagnostic utility in differentiating hepatocellular carcinoma (HCC) from other primary benign or malignant hepatic tumors and from metastatic lesions in the liver. Forty-nine FNAs of the liver obtained between January $\mathbf{2 0 0 0}$ and June 2006 were identified from our cytology files. Cytological diagnoses (confirmed by tissue diagnosis and/or clinical follow-up) included: 7 adenomas, 1 focal nodular hyperplasia (FNH), $24 \mathrm{HCCs}$, and 17 metastatic tumors. On the basis of the histological, clinical and/or radiological follow-up, 20 of 24 (83.3\%) FNAs confirmed positive for HCC-expressed GPC3. All the seven adenomas and the only FNH were negative for GPC3. Sixteen out of seventeen metastatic malignancies were negative for GPC3. The only case expressing GPC3 was an anaplastic carcinoma with neuroendocrine features of unknown origin. In this study, the sensitivity of GPC3 in the diagnosis of HCC in the cytological material was $83.3 \%$, the specificity $96 \%$, the positive predictive value (PPV) $95 \%$ and negative predictive value (NPV) was $85.7 \%$. Immunocytochemical staining for GPC3 in alcohol-fixed FNA material is a highly sensitive and specific method capable of distinguishing $\mathrm{HCC}$ from other benign and malignant hepatic lesions and from the great majority of metastatic lesions.
\end{abstract}

Modern Pathology (2008) 21, 626-631; doi:10.1038/modpathol.2008.26; published online 8 February 2008

Keywords: liver; hepatocellular carcinoma; fine needle aspiration cytology; glypican-3; immunocytochemistry

Early detection of hepatocellular carcinoma (HCC) is very important for the successful treatment of this malignancy. However, differentiating between HCC and benign hepatic lesions is sometimes difficult. ${ }^{1}$ In such a circumstance, a marker that can assist in separating HCC from other hepatic lesions and from metastatic neoplasms to the liver would be very useful.

Glypican-3 (GPC3) a cell surface-linked heparan sulfate proteoglycan that is attached to the cell surface by a glycosyl-phosphatidylinositol anchor is an oncofetal protein that is highly expressed during embryogenesis and organogenesis. It appears to play a role in the regulation of cell proliferation acting through the Wnt signaling pathway. ${ }^{2}$ Specifically, GPC3 is highly expressed in fetal hepatoblasts and is

Correspondence: Dr S Ligato, MD, Department of Pathology and Laboratory Medicine, Hartford Hospital, 80 Seymour Street, Hartford, CT 06102, USA.

E-mail: sligato@harthosp.org

Received 31 May 2007; revised 18 December 2007; accepted 31 December 2007; published online 8 February 2008 absent in most adult tissues including normal adult liver. $^{3}$

Recently, several studies have demonstrated that GPC3 is expressed in both serological ${ }^{4}$ and immunohistochemical material obtained from surgical resections or tissue biopsies of hepatoblastoma and HCC. GPC3 is absent in benign hepatic lesions, cholangiocarcinoma, hepatic cirrhosis and hepatitis. ${ }^{5,6}$

However, to our knowledge, no study has been performed to assess the expression and diagnostic utility of GPC3 in alcohol-fixed cytological material. In this study, we report our experience on the immunocytochemical (ICC) expression of GPC3 in cytological material obtained by FNA of hepatic lesions, and evaluate its diagnostic value in differentiating HCC from other primary and metastatic hepatic lesions.

\section{Materials and methods}

Two hundred and ninety-two FNAs of the liver obtained between January 2000 and June 2006 were 
identified from our cytology files. After review, 243 cases with insufficient/scant cellularity or lack of confirmatory surgical material and/or clinical follow-up were excluded from this study. The remaining 49 cases were selected for GPC3-ICC staining. Cytological diagnoses included: 24 cases of HCC (8 with cirrhosis and 16 with normal liver), 7 adenomas, $1 \mathrm{FNH}$ (focal nodular hyperplasia) and 17 metastatic tumors (3 colorectal carcinomas, 3 pancreatic carcinomas, 2 mammary carcinomas, 2 gastric carcinomas, 1 adenoid cystic carcinoma, 3 non-small cell carcinomas, 1 small cell carcinoma of lung, 1 high grade sarcoma and 1 anaplastic carcinoma with neuroendocrine features). Of the 24 cases of HCC, 7 were nuclear grade (NG) I, 9 NG II and 8 NG III. Alcohol-fixed papanicolaou-stained smears (PAP) were cleared with xylene and rehydrated with graded alcohol to water. Slides were stained with a monoclonal antibody to GPC3 (Clone 1G13, BioMosaics Inc., Burlington, VT, USA). EnVision + polymer (DAKO, Carpinteria, CA) was used for detection. Negative (omission of primary antibody) and positive controls (paraffin sections of HCC) were run in parallel. Optimal primary dilutions were validated on alcohol-fixed cytology slides containing HCC. Results were recorded as negative or positive. GPC3-staining was considered positive when the immunoreactivity was present in at least $3 \%$ of hepatocytes, the extent of reactivity within the neoplastic epithelium was semiquantitatively assessed as focal $(<25 \%$ of positive malignant cells) and diffuse ( $>25 \%$ of positive malignant cell). The intensity was classified as weak, moderate or strong and the location of staining as membranous and cytoplasmic or pericanalicular. An example of focal and weak pattern of staining is illustrated in Figure $3 \mathrm{~b}$.

All cases of malignancy were confirmed based on tissue resection and/or progression of disease on imaging studies. Similarly, lesions were considered benign if there was a lack of progression or resolution on imaging studies in conjunction with continued well-being of the patient. In addition, when available, a diagnosis of benign process made on a resected sample or a tissue biopsy sample from the target area also served to confirm the benign nature of the lesion.

\section{Results}

The results of this study are summarized in Table 1. Based on histological, clinical and/or radiological follow-up 20/24 (83.3\%) FNAs were positive for GPC3 (Figures 1a and b, 2a and b, and 3a and b). All 7 cases of adenoma, (Figure 4a and b) 1 case of $\mathrm{FNH}$ and 16 of the 17 cases of metastatic malignancies (colon carcinoma (0/3), pancreas carcinoma (0/3), breast carcinoma $(0 / 2)$, gastric carcinoma (0/2), adenoid cystic carcinoma (0/1), small cell (0/1), non-small cell carcinomas of lung (0/3) and 0/1 high grade sarcoma) were negative for GPC3. An anaplastic carcinoma with neuroendocrine features of unknown origin was the only case of non-HCC staining for GPC3. In this study, the sensitivity of GPC3 in diagnosing HCC

Table 1 Immunocytochemical expression of glypican-3 in HCC

\begin{tabular}{rlllll}
\hline Case no. & ICC & Nuclear grade & Extent (\%) & Intensity & Distribution \\
\hline 1 & Positive & Grade I & Diffuse (90) & Strong & Cytoplasmic/membrane \\
2 & Positive & Grade I & Diffuse (70) & Strong & Cytoplasmic/membrane \\
3 & Positive & Grade I & Diffuse (100) & Strong & Cytoplasmic/membrane \\
4 & Positive & Grade II & Diffuse (100) & Strong & Cytoplasmic/membrane \\
5 & Negative & Grade III & NA & NA & NA \\
6 & Positive & Grade III & Diffuse (100) & Strong & Cytoplasmic/membrane \\
7 & Positive & Grade II & Diffuse (80) & Moderate & Cytoplasmic/membrane \\
8 & Positive & Grade II & Focal (3) & Weak & Pericanalicular \\
9 & Positive & Grade III & Focal (3) & Weak & Pericanalicular \\
10 & Positive & Grade I & Focal (3) & Weak & Pericanalicular \\
11 & Positive & Grade I & Diffuse (100) & Strong & Cytoplasmic/membrane \\
12 & Positive & Grade I & Diffuse (90) & Moderate & Cytoplasmic/membrane \\
13 & Positive & Grade III & Focal (4) & Weak & Pericanalicular \\
14 & Negative & Grade III & NA & NA & NA \\
15 & Positive & Grade II & Diffuse (80) & Strong & Cytoplasmic/membrane \\
16 & Positive & Grade II & Diffuse (70) & Strong & Cytoplasmic/membrane \\
17 & Negative & Grade II & NA & NA & NA \\
18 & Positive & Grade II & Diffuse (90) & Strong & Cytoplasmic/membrane \\
19 & Positive & Grade III & Diffuse (80) & Strong & Cytoplasmic/membrane \\
20 & Negative & Grade III & NA & NA & NA \\
21 & Positive & Grade II & Diffuse (90) & Strong & Cytoplasmic/memb \\
22 & Positive & Grade II & Focal (3) & Weak & Pericanalicular \\
23 & Positive & Grade I & Diffuse (80) & Moderate & Cytoplasmic/memb \\
24 & Positive & Grade III & Focal (3) & Moderate & Cytoplasmic/membrane \\
& & & & Absent \\
& & & & Absent \\
\end{tabular}

NA, not applicable. 

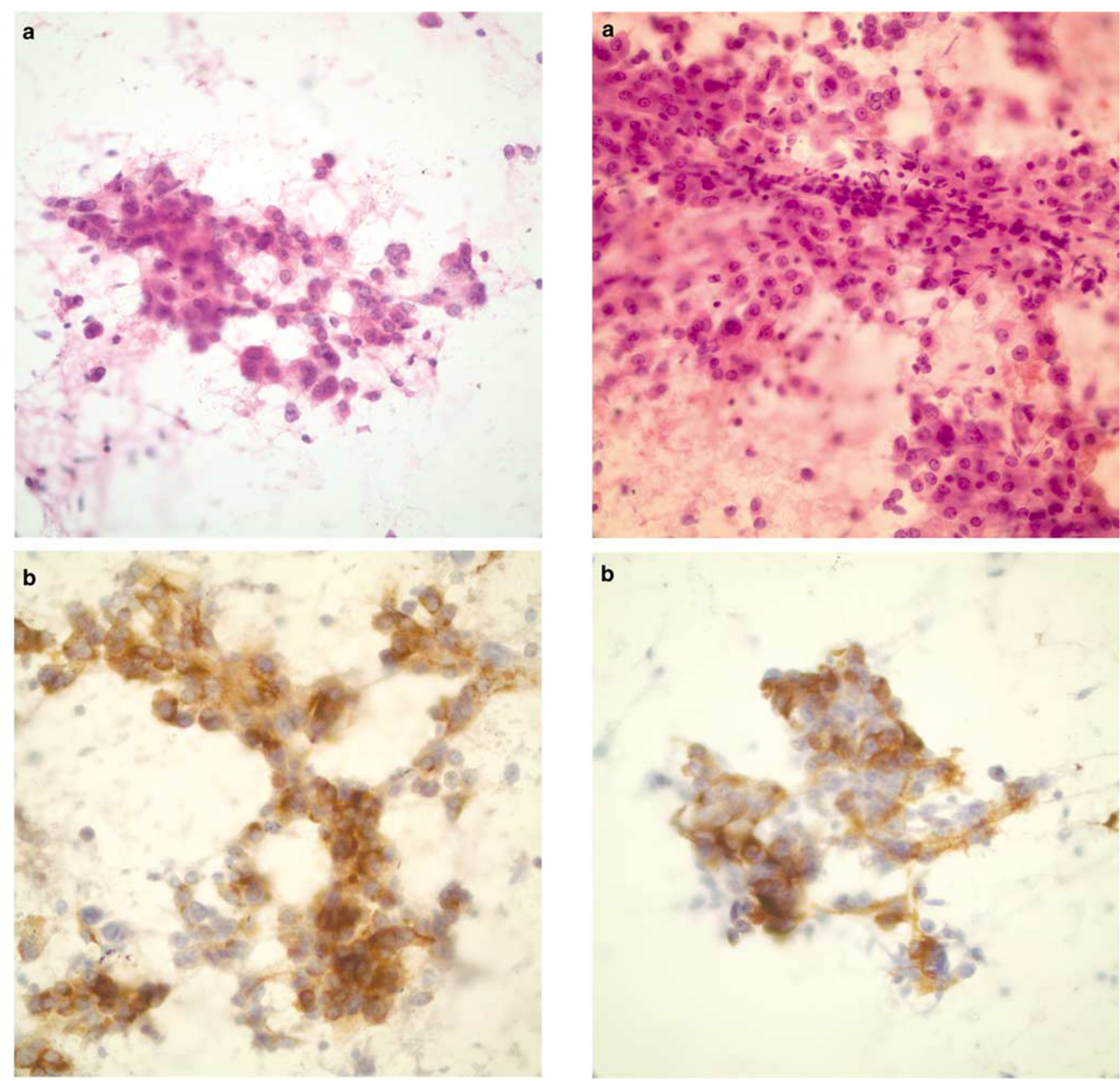

Figure 1 (a) FNA of HCC nuclear grade (NG) I. $(\times 400$, PAP stained). (b) Positive ICC stain for GPC3 in HCC NG I with a strong cytoplasmic and membrane-based pattern of staining (original magnification $\times 400$ ).

was $83.3 \%$, the specificity $96 \%$, the PPV $95 \%$ and an NPV of $85.7 \%$. (Table 2).

As summarized in Table 1, the extent of staining in the 20 HCCs expressing GPC3 was diffuse in 14 cases $(70 \%)$, and focal in 6 cases $(30 \%)$. The intensity of staining was weak in 5 cases $(25 \%)$, moderate in 4 cases $(20 \%)$ and strong in 11 cases $(55 \%)$. The distribution of staining was membranous and cytoplasmic-based with a diffuse, uniform granular pattern in 15 cases $(75 \%)$ and pericanalicular in 5 cases (25\%) (Figures $1 \mathrm{~b}, 2 \mathrm{~b}$ and $3 \mathrm{~b}$ ). In the

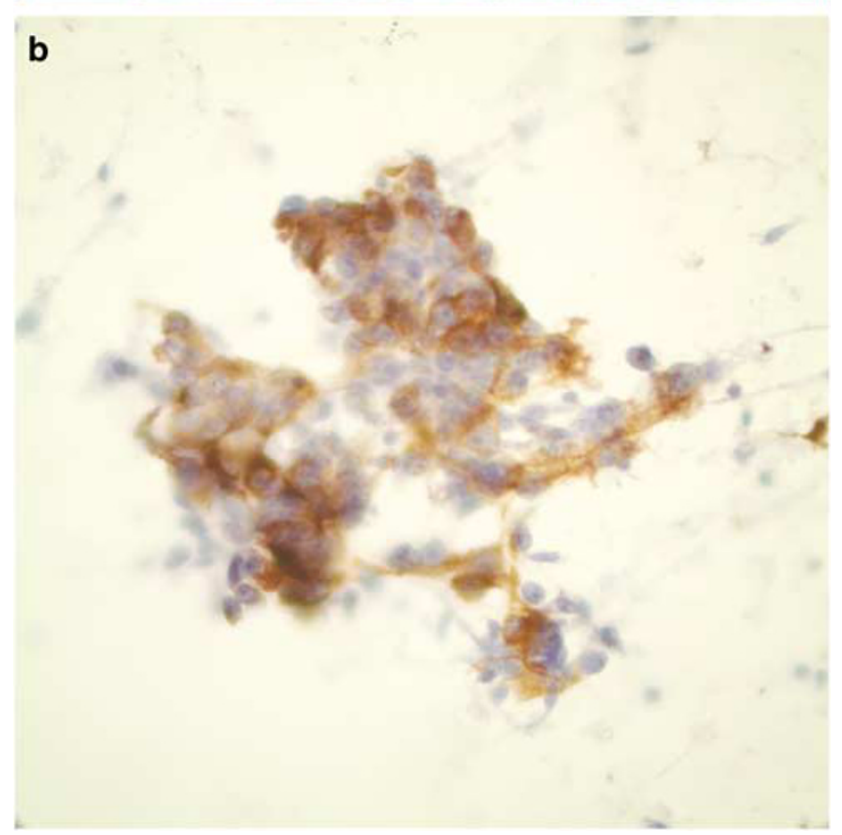

Figure 2 (a) FNA of HCC NG II ( $\times$ 400, PAP stained). (b) Positive ICC stain for GPC3 in HCC NG II with a moderate, prevalently membranous pattern of staining (original magnification $\times 400$ ).

only case of non-HCC positive for GPC3 the staining was focal (with only $3 \%$ of the cells positive), with a weak intensity and a pericanalicular pattern of distribution. No positive staining for GPC3 was observed in bland/normal-appearing hepatocytes or biliary ductal cells. Six of $8(75 \%)$ HCCs with cirrhosis were positive for GPC3, whereas 14 of 16 $(87.5 \%)$ HCCs without cirrhosis expressed GPC3. Of the four cases of HCC that did not expresse GPC3, two cases were without evidence of cirrhosis and the other two cases were cirrhotic. 

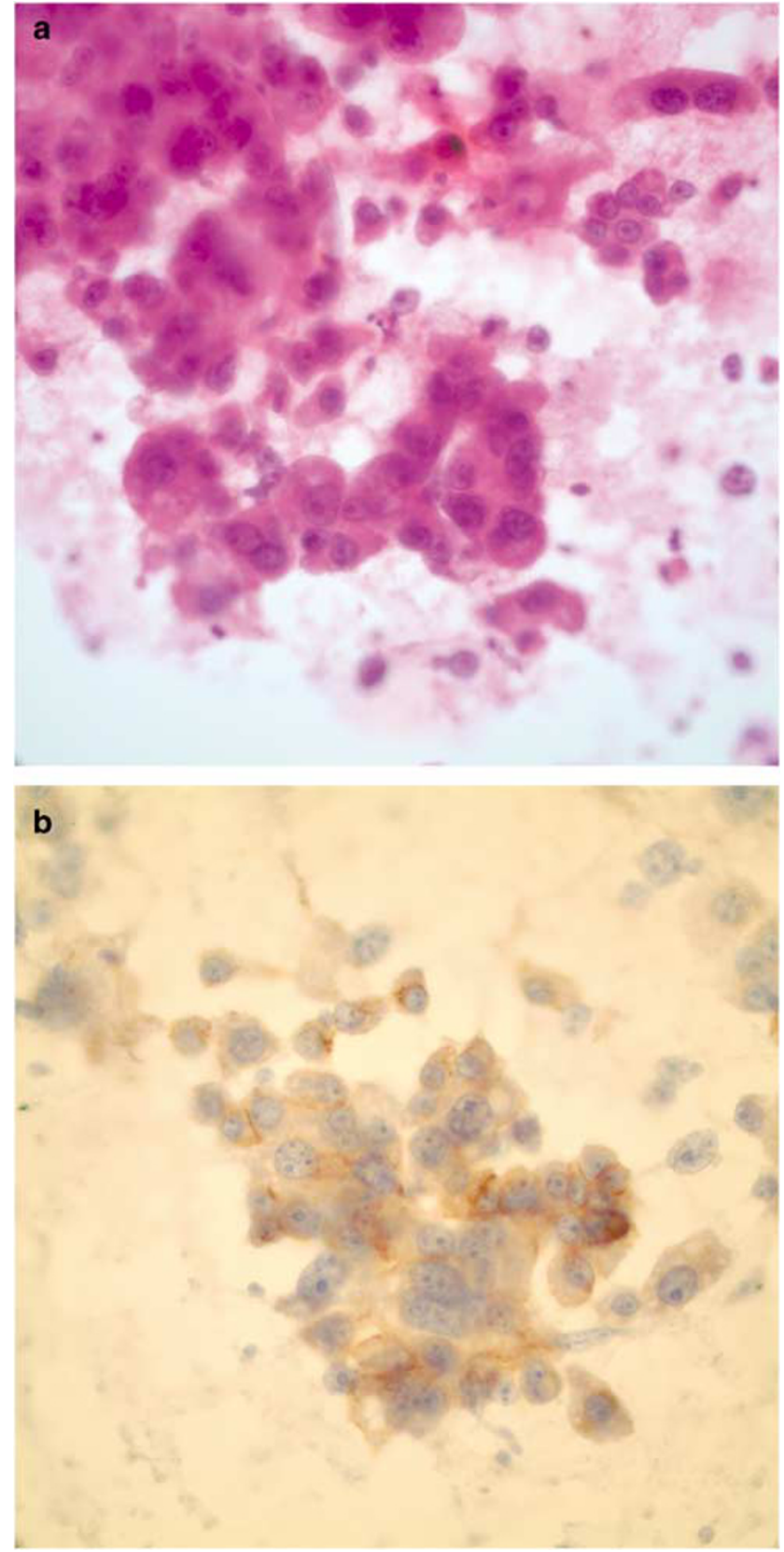

Figure 3 (a) FNA of HCC NG III. ( $\times$ 400, PAP stained). (b) Positive ICC stain for GPC3 in HCC NG III with only a weak and focal pattern of staining. Note the pericanalicular pattern of staining of the malignant cells. (original magnification $\times 400$ ).

\section{Discussion}

Several recent studies have identified that GPC3, used as a single immunohistochemical $\operatorname{stain}^{3-9}$ or as part of a panel with other markers such as HSP70, and glutamine synthetase ${ }^{10}$ may be very helpful in differentiating HCC from benign hepatic lesions and from metastatic neoplasms to the liver.

These studies, performed on formalin-fixed paraffin-embedded tissues, have demonstrated that GPC3 is not expressed in hepatocytes from normal liver and benign hepatic lesions such as adenoma,

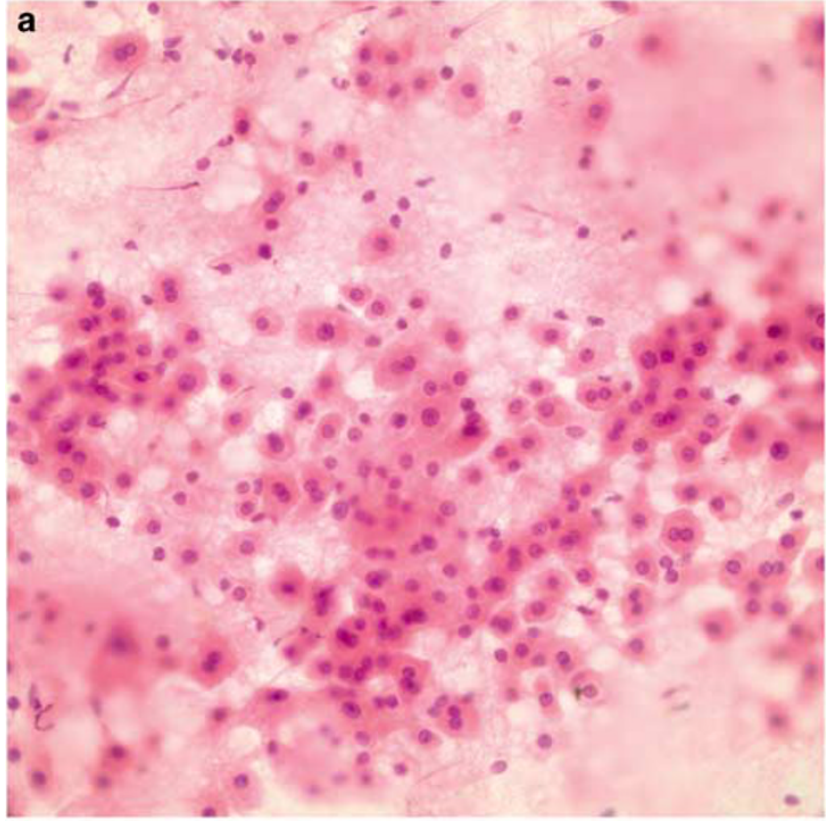

b

Figure 4 (a) FNA of adenoma. ( $\times$ 400, PAP stained). (b) Negative ICC stain for GPC3 in adenoma cells. (original magnification $\times 400)$.

FNH, cirrhosis, hepatitis ${ }^{4}$ and FNH-like nodules in cirrhotic liver; ${ }^{8}$ and is expressed in the majority of cases of HCC with a sensitivity ranging from 72 to $90 \%{ }^{3,4,8,9}$ and a specificity between 96 and $100 \%{ }^{8}$

In addition, some of these studies have identified that hepatic preneoplastic lesions such as high grade dysplastic nodules (HGDNs) can express GPC3 in appoximately $7 \%$ of the cases; and low grade dysplastic/large regenerative nodules express GPC3 in only $3 \%$ of the cases. ${ }^{8}$

To our knowledge, this is the first study that has investigated the ICC expression of GPC3 in 
Table 2 Results of sensitivity, specificity, positive predictive value (PPV) and negative predictive value (NPV) of GPC3 ICC in HCC

\begin{tabular}{lcccc}
\hline ICC & Sensitivity & Specificity & PPV & NPV \\
\hline glypican-3 & $83.3 \%$ & $96 \%$ & $95 \%$ & $85.7 \%$ \\
\hline
\end{tabular}

Table 3 Relation between nuclear grade (NG) of HCC and GPC3 expression

\begin{tabular}{llccc}
\hline No. of cases & GPC-3 ICC Stain & NG I & NG II & NG III \\
\hline HCCs 20 & Positive & 7 & 8 & 5 \\
HCCs 4 & Negative & 0 & 1 & 3 \\
Total 24 & - & 7 & 9 & 8 \\
\hline
\end{tabular}

alcohol-fixed cytological material obtained by FNA of the liver. Our data indicated that GPC3 is expressed in the majority of cytological specimens of HCC with a sensitivity of $83.3 \%$ and a specificity of $96 \%$, similar to the sensitivity and specificity observed in several studies performed in formalinfixed paraffin-embedded tissue., ${ }^{2,3,8,9}$

Furthermore, we observed similar membranous/ cytoplasmic and pericanalicular patterns of staining for GPC3 as previously documented in tissue samples. ${ }^{3,9}$ In fact, $75 \%$ of our cases of HCC positive for GPC3 showed a membranous/cytoplasmic-based pattern of staining (Figures $1 \mathrm{~b}$ and $2 \mathrm{~b}$ ) and $25 \%$ a pericanalicular pattern (Figure $3 \mathrm{~b}$ ).

In addition, a possible correlation between the extent, intensity and distribution of staining of GPC3 in HCC was noted. In fact, five of the six cases of HCC that showed a focal extent of staining were also associated with a weak intensity of staining and a prevalently pericanalicular pattern. (Table 1).

As summarized in Table 3, we noticed that as the NG of HCC increases the expression of GPC3 decreases. In fact, GPC3 was expressed in all seven cases $(100 \%)$ of HCC with NG I, eight of nine cases $(88.9 \%)$ with NG II and five of eight cases (62.5\%) with NG III. In this respect, our results differ from the findings reported in other studies performed in tissue sections in which a higher number of HCCs with NG II and III (approximately 82\%) were positive for GPC3 compared to well differentiated HCCs with NG I (53\%). ${ }^{9}$

In our study, $75 \%$ (6/8) of HCC from cirrhotic livers expressed GPC3 compared with 87.5\% (14/16) of HCC developing in normal livers. This finding differs from the recent observation reported by Wang et $a .^{9}$ in a study performed in tissue samples, where a statistically significant correlation between GPC3 expression in HCC from cirrhotic livers (90\%) compared with HCC in normal livers (64\%),
$P<0.01$ was found. We do not have an explanation for these differences, and only additional studies performed in cytological material will help to clarifying these discrepancies.

As our and other studies have shown, it appears that GPC3 is not expressed in approximately $25-30 \%$ of tissue samples of HCC and in $16.7 \%$ of our cytological smears. One reason could be attributed to the heterogeneous molecular pathways through which hepatocytes may progress during the process of hepatocarcinogenesis. Another explanation could be related to the focal expression of GPC3 in HCC. ${ }^{8}$

Therefore, there is a certain risk of sampling error particularly in cytological material, and consequently negative results do not always exclude a diagnosis of HCC. On the other hand, we caution to interpret a positive ICC stain for GPC3 as a definitive proof for a diagnosis of HCC. In fact, due to the focal expression of GPC3 in dysplastic nodules, and in rare cases, in metastatic carcinomas to the liver, ${ }^{11}$ a positive result for GPC3 in non-HCC may be obtained.

Therefore, it is prudent to interpret a positive ICC result for GPC3 within the appropriate clinical, serological and radiological context.

Our results indicate that GPC3 is expressed in the majority of cases of HCC obtained by FNA of the liver with a diagnostic accuracy similar to the one already documented in formalin-fixed tissue. These findings suggest that fixation in alcohol and subsequent PAP staining does not affect the ICC detection of the GPC3.

In conclusion, we have demonstrated that ICC staining for GPC3 in alcohol-fixed cytological material is a valuable tool capable of differentiating HCC from other hepatic lesions, and from most of the metastatic tumors to the liver. Since GPC3 ICC stain is simple to interpret and can be performed in most laboratories currently performing ICC on cytological specimens, the routine use of this marker in clinical practice may become a valuable diagnostic adjunct to the cytological diagnosis of HCC.

\section{References}

1 Wee A, Nilsson B, Tan LKA, et al. Fine needle aspiration biopsy of hepatocellular carcinoma: diagnostic dilemma at the ends of the spectrum. Acta Cytolol 1994;38:347-354.

2 Capurro M, Xiang Y, Lobe C, et al. Glypican-3 promotes the growth of hepatocellular carcinoma by stimulating canonical Wnt signaling. Cancer Research 2005;65:6245-6254.

3 Yamauchi N, Watanabe A, Hishinuma M, et al. The glypican 3 oncofetal protein is a promising diagnostic marker for hepatocellular carcinoma. Mod Pathol 2005;18:1591-1598.

4 Capurro M, Wanless IR, Sherman M, et al. Glypican-3: a novel serum and histochemical marker for hepatocellular carcinoma. Gastroenterology 2003;125:89-97. 
5 Filmus J, Capurro M. Glypican-3 and alpha-fetoprotein as diagnostic tests for hepatocellular carcinoma. Mol Diagn 2004;8:207-212.

6 Man XB, Tang L, Zhang BH, et al. Upregulation of Glypican-3 expression in hepatocellular carcinoma but downregulation in cholangiocarcinoma indicates its differential diagnosis value in primary liver cancers. Liver Int 2005;25:962-966.

7 Zhu ZW, Friess H, Wang L, et al. Enhanced glypican-3 expression differentiates the majority of hepatocellular carcinomas from benign hepatic disorders. Gut 2001; 48:558-564.

8 Libbrecht L, Severi T, Cassiman D, et al. Glypican-3 expression distinguishes small hepatocellular carcinomas from cirrhosis, dysplastic nodules, and focal nodular hyperplasia-like nodules. Am J Surg Path 2006;30: 1405-1411.

9 Wang YX, Degos F, Dubois S, et al. Glypican-3 expression in hepatocellular tumors: diagnostic value for preneoplastic lesions and hepatocellular carcinomas. Hum Path 2006;37:1435-1441.

10 DiTommaso L, Franchi G, Park YN, et al. Diagnostic Value of HSP70, Glypican 3, and Glutamine Synthetase in Hepatocellular Nodules in Cirrhosis. Hepatology 2007;45:725-734.

11 Jia AY, Hwang YQ, Allegretta M, et al. Abstract \#561 presented at the XXVI Congress of the IAP. Mod Path 2006;123. 